\title{
Hepatic Insulin Responsiveness in Patients with Endogenous Hypertriglyceridaemia
}

\author{
R. M. Bernstein, B. M. Davis, J. M. Olefsky, and G. M. Reaven \\ Department of Medicine, Stanford University School of Medicine and Veterans Administration Hospital, Palo Alto, California, USA
}

\begin{abstract}
Summary. Plasma insulin response to oral glucose, insulin resistance, and insulin suppression of hepatic glucose production were studied in 11 normal subjects and 11 hypertriglyceridaemic patients. Patients with hypertriglyceridaemia had a significantly higher insulin response to oral glucose. Insulin resistance was also significantly greater in hypertriglyceridaemic subjects as determined by measuring the steady-state plasma glucose response during a continuous infusion of epinephrine, propranolol, glucose, and exogenous insulin. Insulin suppression of hepatic glucose production was calculated from the results of two studies in which glucose turnover rate was measured by a continuous infusion of ${ }^{3} \mathrm{H}-2$-glucose. The first study was performed under conditions of basal insulin secretion, and the second carried out at steady state exogenous insulin levels of approximately $100 \mu \mathrm{U} / \mathrm{ml}$. The results indicated that basal hepatic glucose production was the same in both groups, and was suppressed to an equal degree by physiological levels of insulin. These data demonstrate that hepatic glucose production can be suppressed to an equal degree in normal and hypertriglyceridaemic subjects at comparable circulating insulin levels, at the same time that resistance to insulin-stimulated glucose uptake is observed in the hypertriglyceridaemic individuals.
\end{abstract}

Key words: Hypertriglyceridaemia, insulin resistance, hyperinsulinaemia, hepatic glucose output, triglyceride metabolism.

Several years ago we formulated a pathogenetic scheme for the development of endogenous hypertriglyceridaemia which we felt to be present in most patients with this disorder $[1,2]$. The proposed se- quence of events is as follows: resistance to glucose uptake $\rightarrow$ hyperinsulinaemia $\rightarrow$ increased hepatic production of very low density lipoprotein triglyceride (VLDL-TG) $\rightarrow$ increased plasma triglyceride levels. The cumulative evidence in support of this overall hypothesis has been reconsidered recently [3], and we again concluded that hyperinsulinaemia plays an important role in modulation of hepatic triglyceride production.

Implicit in the above scheme is the assumption that the liver of an insulin resistant hypertriglyceridaemic subject can still show insulin stimulation of hepatic triglyceride synthesis [4-7]. This assumption has been criticized on the grounds that if insulin resistance is a primary defect in patients with endogenous hypertriglyceridaemia, then insulin resistance should be present in all tissues and hyperinsulinaemia could not stimulate hepatic VLDL-TG synthesis and secretion in these patients $[8,9]$. However, no direct evidence is available concerning hepatic responsiveness to insulin in patients with endogenous hypertriglyceridaemia. In order to provide such data we carried out the present study, in which several facets of insulin action have been compared in patients with normal or elevated plasma triglyceride levels.

\section{Materials and Methods}

\section{Subjects}

Twenty-two non-obese subjects with fasting plasma glucose levels of less than $110 \mathrm{mg} / 100 \mathrm{ml}$ were studied after informed consent had been obtained. All patients consumed a weight maintenance liquid formula diet, consisting of $43 \%$ carbohydrate, $42 \%$ fat, and $15 \%$ protein. The daily caloric intake of $35 \mathrm{Kcal} /$ $\mathrm{kg} /$ day was divided into portions of $1 / 5,2 / 5$ and $2 / 5$ 


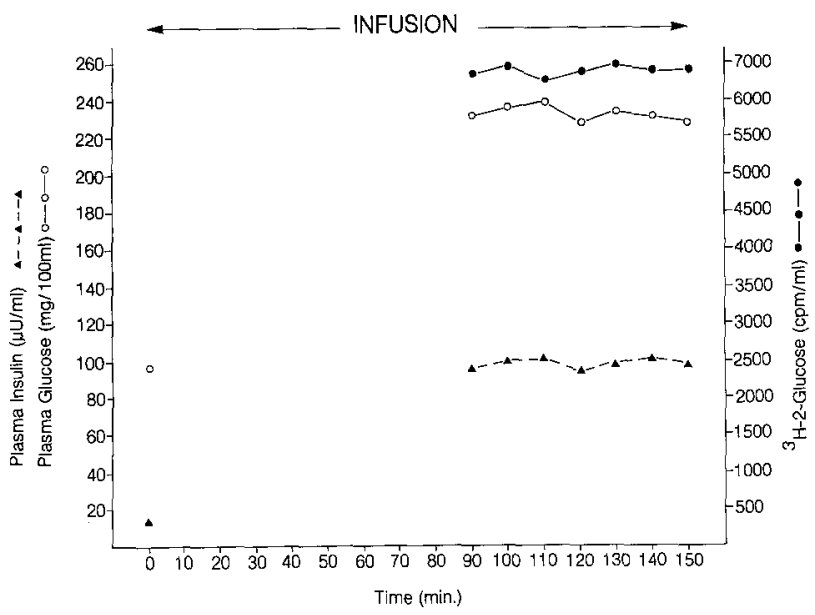

Fig. 1. Experimental results obtained during one insulin infusion study in a hypertriglyceridaemic patient. The infusion contained insulin $(80 \mathrm{mU} / \mathrm{min})$, epinephrine $(6 \mu \mathrm{g} / \mathrm{min})$, propranolol $(0.08$ $\mathrm{mg} / \mathrm{min})$, glucose $(6 \mathrm{mg} / \mathrm{kg} / \mathrm{min})$ and ${ }^{3} \mathrm{H}-2$ glucose $(1.67 \mu \mathrm{Ci} / \mathrm{min})$

served at $8 \mathrm{AM}, 12$ Noon and $6 \mathrm{PM}$. Daily weights were obtained on all subjects and were constant throughout the study period. There was an initial three day period of dietary equilibration before studies were begun. A subject's mean fasting triglyceride level was calculated from at least four separate triglyceride measurements made during the week following the period of stabilization. Subjects were considered normal if their mean fasting triglyceride value was $\leqslant 125 \mathrm{mg} / 100 \mathrm{ml}$. Subjects were considered to have hypertriglyceridaemia if their mean fasting triglyceride value was $\geqslant 175 \mathrm{mg} / 100 \mathrm{ml}$. Patients with plasma triglyceride levels between these two values were not studied. On this basis we selected 22 patients for study; 11 normal and 11 with hypertriglyceridaemia. They were all in good general health, without evidence of hepatic or cardiac disease. None of the patients had secondary hyperlipaemia, and none were taking any medications known to affect carbohydrate or lipid metabolism.

\section{Protocol}

After the period of dietary stabilization, an oral glucose tolerance test (OGTT) was performed using $40 \mathrm{gm}$ of glucose per sq. metre body surface. One week later glucose turnover rate was determined after an overnight fast by a continuous infusion technique, utilizing ${ }^{3} \mathrm{H}-2$-glucose $(5.8 \mathrm{Ci} / \mathrm{mM})$. One hundred fifty $\mu \mathrm{Ci}$ of ${ }^{3} \mathrm{H}-2$-glucose were given by continuous infusion for $150 \mathrm{~min}$, and blood withdrawn $120,125,130,135,140,145$ and 150 minutes for determination of glucose radioactivity and measurement of plasma glucose and insulin levels. This was termed the basal study, and permits calculation of hepatic glucose production in the fasting state.

The glucose turnover measurement was repeated several days later, but on this occasion insulin resistance was also determined. Thus, in addition to the ${ }^{3} \mathrm{H}$-2-glucose $(250 \mu \mathrm{Ci})$, the infusion mixture also contained unlabelled glucose $(6 \mathrm{mg} / \mathrm{kg} / \mathrm{min})$, insulin $(80 \mathrm{mU} / \mathrm{min})$, epinephrine $(6 \mathrm{\mu g} / \mathrm{min})$ and propranolol $(0.08 \mathrm{mg} / \mathrm{min})$. This was termed the insulin infusion study. Under these experimental conditions, endogenous insulin secretion is inhibited and steady state plasma glucose (SSPG) and exogenous insulin (SSPI) levels are reached by 90 minutes. As before, blood was drawn for determination of glucose radioactivity and plasma glucose and insulin levels every 5 minutes during the last 30 minutes of the 150 minute infusion period. This study determined the ability of identical amounts of insulin to limit the magnitude of the steady state plasma glucose response in various subjects (insulin resistance), as well as the measurement of hepatic glucose production during the insulin infusion. By comparing the values for hepatic glucose production in each subject during these two studies, the ability of insulin to suppress hepatic glucose production in normal as compared to hypertriglyceridaemic subjects could be estimated.

Studies in which the coefficients of variation of the individual steady state plasma glucose, steady state plasma insulin or ${ }^{3} \mathrm{H}$-2-glucose values were greater than $10 \%$ were excluded. The actual data obtained during one infusion study are plotted in Figure 1.

\section{Analytical Methods}

Blood for determination of plasma glucose, insulin, tritiated glucose and triglyceride was drawn into test tubes containing $14 \mathrm{mg}$ of EDTA and placed in the cold. Plasma was separated within 15 minutes and aliquots stored at $-20^{\circ} \mathrm{C}$. Plasma glucose was measured by an automated glucose oxidase method (Beckman Instruments. Fullerton, Cal.), plasma insulin by the method of Desbuquois and Aurbach [10] and plasma triglyceride levels by a modification of the method of Kessler and Lederer [11]. The radioactivity of tritiated glucose was determined by the method of Katz and Dunn [12].

\section{Calculations}

Hepatic Glucose Production (HGP): Both the liver and the kidney are capable of producing and releasing glucose into the plasma. Although glucose production by the kidney may become significant during prolonged fasting, current evidence strongly suggests 
that the renal contribution to the plasma glucose pool is insignificant in the postabsorptive state $[13,14]$. Therefore, under the conditions of this study it seems reasonable to assume that during the basal study the liver is the only appreciable source of glucose. In this situation, hepatic glucose production is equal to glucose turnover rate. Thus, HGP = glucose turnover $(\mathrm{mg} / \mathrm{min})=$

$\frac{\text { tritiated glucose infusion rate (cts } / \mathrm{min} \text { ) }}{\text { specific activity of plasma glucose (cts } / \mathrm{mg} \text { ). }}$

During the insulin infusion study, however, glucose turnover is determined while subjects are receiving a constant infusion of exogenous glucose $(6 \mathrm{mg} / \mathrm{kg} /$ min) and this must be subtracted from the total glucose turnover rate in order to obtain HGP. In this study, $\mathrm{HGP}=$ glucose turnover rate $(\mathrm{mg} / \mathrm{kg} / \mathrm{min})$ infusion rate $(6 \mathrm{mg} / \mathrm{kg} / \mathrm{min})$.

\section{Statistical Analysis}

All results are expressed as the mean \pm SEM. Student's t-test was used to compare the differences between the two groups.

\section{Results}

Nine of the eleven normal patients were males, as compared to ten males among patients with hypertriglyceridaemia. Normal subjects had a mean age of 45 \pm 3 , with a range from $36-59$ years, as compared to hypertriglyceridaemic patients whose mean age was $47 \pm 3$, with a range from 32-61 years. The mean relative weight of the normal subjects was $0.92 \pm$ 0.03 and this was not significantly different from a mean of $1.00 \pm 0.03$ for the hypertriglyceridaemic patients. However, there was considerable difference between the mean triglyceride levels of the two groups, being $100 \pm 8 \mathrm{mg} / 100 \mathrm{ml}$ and $293 \pm 44 \mathrm{mg} /$ $100 \mathrm{ml}$ in the normal and hypertriglyceridaemic subjects, respectively. Thus, a difference in the triglyceride levels of the two groups of subjects was present in the absence of differences in age, sex, or degree of obesity.

Mean plasma glucose $(\mathrm{mg} / 100 \mathrm{ml})$ and insulin $(\mu \mathrm{U} / \mathrm{ml})$ responses of the two groups to oral glucose are shown in Figure 2. There were no significant differences in the plasma glucose responses of the two patient groups. It is noteworthy that the mean insulin response of the hypertriglyceridaemic group was higher at all points when compared to the normal subjects. These differences were statistically significant $(\mathrm{p}<0.05)$ before the oral glucose load and at one and two hours after this load.

The estimates of insulin resistance in the two

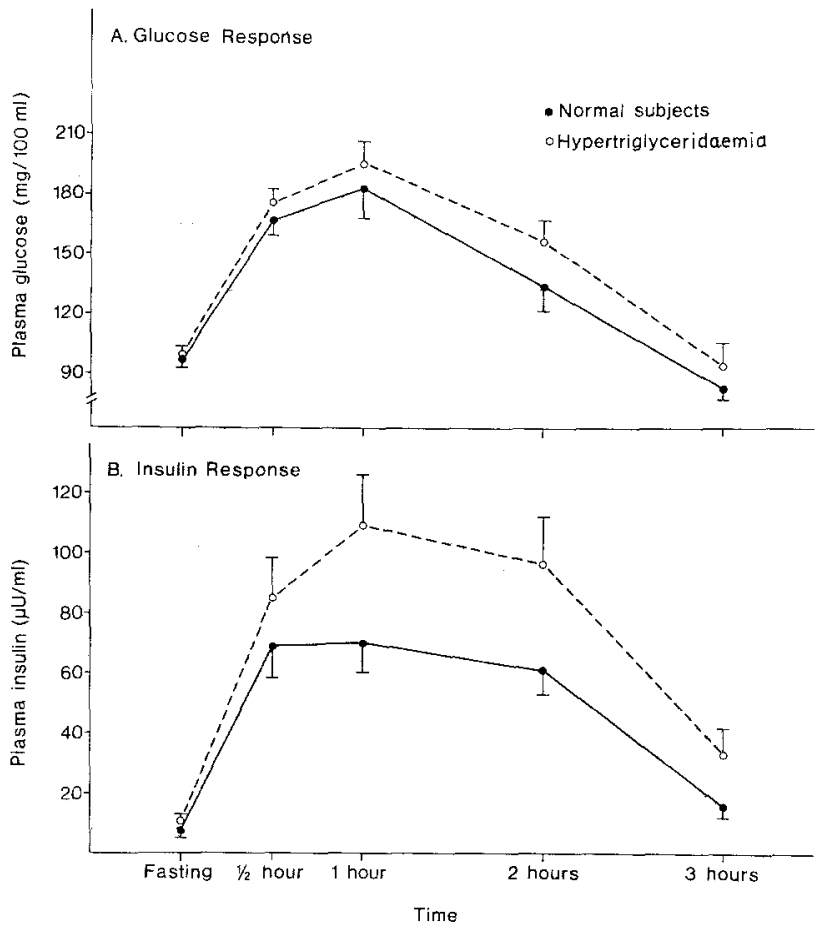

Fig. 2. Mean ( \pm SEM) plasma glucose and insulin responses of normal and hypertriglyceridaemic subjects during an oral glucose tolerance test $(40 \mathrm{~g} /$ square meter body surface area)

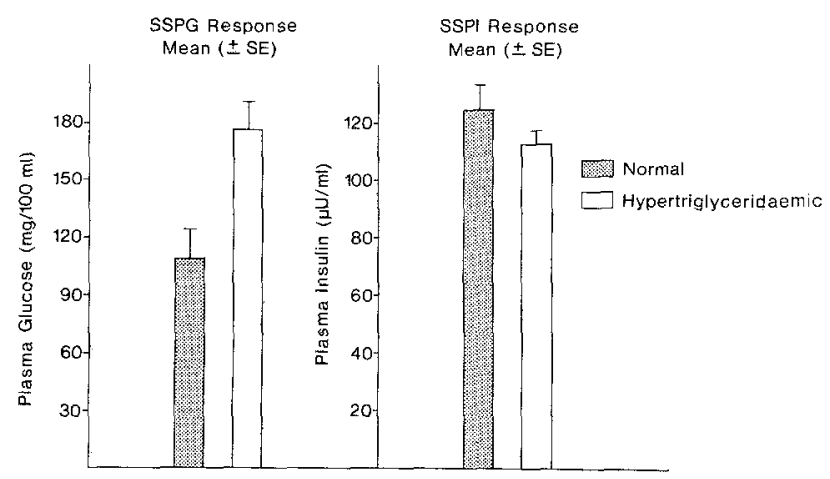

Fig. 3. Mean ( \pm SEM) steady state plasma glucose (SSPG) and insulin (SSPI) responses during the infusion of epinephrine, propranolol, glucose and exogenous insulin

groups are shown in Figure 3. The mean steady state plasma glucose response of the hypertriglyceridaemic patients was significantly greater $(p<0.01)$ than that of the control subjects. Since steady state plasma insulin levels were the same in both groups, it is clear that the patients with hypertriglyceridaemia were more resistant to the ability of insulin to promote disposal of the glucose load.

Figure 3 shows that there was no difference in the hepatic glucose production (HGP) of the two experimental groups during the basal and infusion studies. 
EFFECT OF INSULIN INFUSION ON HEPATIC GLUCOSE PRODUCTION (HGP)

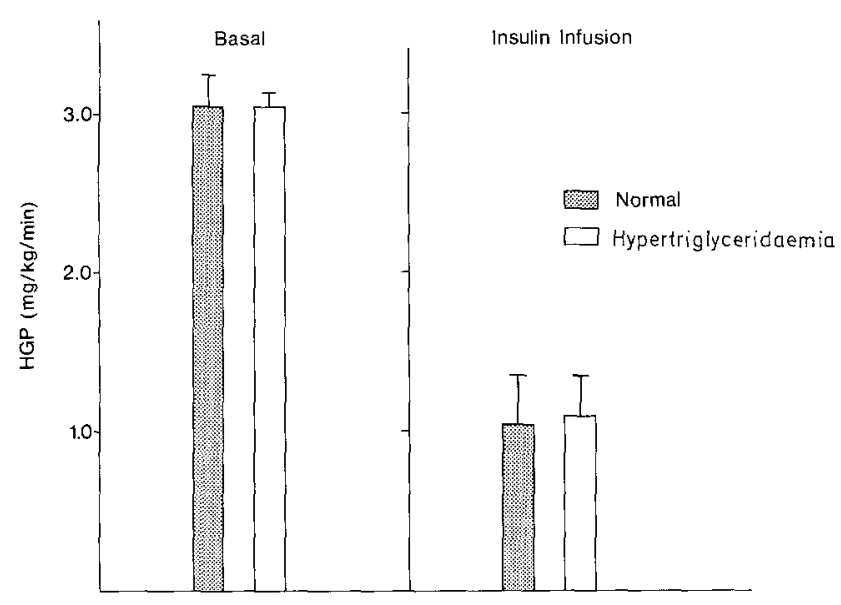

Fig. 4. Mean ( \pm SEM) hepatic glucose production (HGP) in normal and hypertriglyceridaemic subjects under basal conditions (left panel) and during the infusion of epinephrine, propranolol, glucose and exogenous insulin (right panel)

However, it should be emphasized that these results were only obtained at one insulin level, albeit at the same insulin concentration used to demonstrate the presence of resistance to insulin-stimulated glucose uptake.

\section{Discussion}

These results reaffirm that patients with endogenous hypertriglyceridaemia are hyperinsulinaemic and insulin resistant when compared to a group of control subjects of comparable age and degree of obesity (1-3). The insulin resistance of these patients has been demonstrated by measuring the ability of comparable circulating levels of exogenous insulin to limit the degree of hyperglycaemia attained during the administration of a continuous glucose load. The higher steady state plasma glucose levels that were reached in the hypertriglyceridaemic patients could result from greater resistance to either insulin suppression of hepatic glucose production and/or insulin stimulation of glucose uptake. In addition, the higher steady state plasma glucose levels in the hypertriglyceridaemic subjects could be due to the fact that their glucose transport system has become saturated at the higher plasma glucose levels. The first of these alternatives seems the least likely, given the fact that hepatic glucose production was suppressed to an equal degree in both experimental groups. The data available do not permit any conclusions as to which of the two other theoretical alternatives is primarily responsible for the higher SSPG levels in hypertriglyceridaemic patients.
Although these results indicate that patients with endogenous hypertriglyceridaemia were as responsive as normal subjects to insulin suppression of hepatic glucose production at steady state insulin levels of approximately $100 \mu \mathrm{U} / \mathrm{ml}$, this does not imply that their livers retain normal insulin sensitivity. For example, it is certainly possible that circulating insulin levels of $25 \mu \mathrm{U} / \mathrm{ml}$ might well suppress hepatic glucose production to a greater degree in normal subjects. However, the goal of the present study was not to determine the relative ability of varying doses of circulating insulin to suppress hepatic glucose production in normal versus hypertriglyceridaemic subjects. Rather, our aim was to see if there was a dissociation between the ability of insulin to stimulate glucose disposal as contrasted to its ability to suppress hepatic glucose production, and to make this comparison at a physiological level of insulin. In order to do this we achieved steady state plasma insulin levels of approximately $100 \mu \mathrm{U} / \mathrm{ml}$, concentrations that are within the range seen in the peripheral plasma following a glucose challenge. Indeed, the insulin level is significantly less than would exist in the portal vein at the same time. Given these considerations, we think it possible to conclude that the ability of insulin to suppress hepatic glucose production is comparable in normal subjects and patients with endogenous hypertriglyceridaemia at insulin concentrations which commonly exist in the portal vein, and at insulin levels which result in different rates of glucose disposal in the two groups.

Finally, in order to put these conclusions into perspective, several qualifications must be made explicit. In the first place, the crucial observations were made during the continuous infusion of epinephrine, propranolol, glucose and insulin. It is certainly possible that differential effects of epinephrine and propranolol in the two patient groups could account for the experimental observations. Thus, the epinephrine and propranolol could have both interfered with the ability of insulin to stimulate glucose disposal, and/or potentiated the ability of insulin to inhibit hepatic glucose production in patients with hypertriglyceridaemia. Although these disparate pharmacological effects could account for the observed differences, this does seem somewhat unlikely, particularly in light of the known effects of epinephrine on the liver. Secondly, it must be remembered that we have assumed that essentially all the new glucose that enters the circulation comes from the liver, and that the kidney contribution is nil under the conditions of our studies. Although we feel that the available evidence $[13,14]$ solidly supports this view, we did not directly measure the contribution of the kidney to total glucose production. 
Lastly, it must be emphasized that these results do not prove that the livers of patients with endogenous hypertriglyceridaemia respond to their endogenous hyperinsulinaemia by increasing hepatic VLDL-TG synthesis and secretion. However, given the fact that the livers of these patients respond to at least one action of insulin at physiological levels, it is at least possible to suggest that other hepatic actions of insulin may also be maintained.

Acknowledgements. This work was supported in part by a grant from the National Institutes of Health, HL 08506, and from the General Clinical Research Centers Branch, \#RR-70, and from the Research Services of the Veterans Administration.

\section{References}

1. Farquhar, J. W., Frank, A., Gross, R. C., Reaven, G. M.: Glucose, insulin and triglyceride responses to high and low carbohydrate diets in man. J. Clin. Invest. 45, 1648-1565 (1966)

2. Reaven, G.M., Lerner, R.L., Stern, M.P., Farquhar, J.W.: Role of insulin in endogenous hypertriglyceridemia. J. Clin. Invest. 46, 1756-1767 (1967)

3. Olefsky, J.M., Farquhar, J. W., Reaven, G. M.: Reappraisal of the role of insulin in hypertriglyceridemia. Am. J. Med. 57, 551-560 (1974)

4. Salans, L. B., Reaven, G. M.: Effects of insulin pretreatment on glucose and lipid metabolism of liver slices from normal rats. Proc. Soc. Exp. Biol. Med. 122, 1208-1213 (1966)

5. Letarte, J., Fraser, T. R.: Stimulation by insulin of the incorporation of $\mathrm{U}^{14} \mathrm{C}$-glucose into lipids released by the liver. Diabetologia 5, 358-359 (1969)

6. Topping, D.L., Mayes, P. A.: The immediate effects of insulin and fructose on the metabolism of the perfused liver. Biochem. J. 120, 295-311 (1972)

7. Tulloch, B. R., Dyal, K., Fraser, T. R.: Increased lipid synthesis by liver slice in a superfusion system following raised glucose or insulin concentration. Diabetologia 8, 267-273 (1972)

8. Havel, R.: Lipid transport and insulin resistance. Excerpta Med. 20, 353-359 (1973)

9. Kissebah, A. H., Alfarsi, S., Adams, P. W., Wynn, V.: Role of insulin resistance in adipose tissue and liver in the pathogenesis of endogenous hypertriglyceridaemia in man. Diabetologia 12, 563-571 (1976)

10. Desbuquois, B., Aurbach, G. D.: Use of polyethylene glycol to separate free and antibody-bound peptide hormones in radioimmunoassay. J. Clin. Endocrinol. Metab. 33, 732-738 (1971)

11. Kessler, G., Lederer, H.: Fluorometric measurement of triglyceride. In: L. T. Skeggs, Jr. (Ed.): Automation in Analytic Chemistry, pp. 341-344. New York: Med. Incorp. 1976

12. Katz, J., Dunn, A.: Glucose-2-T as a tracer for glucose metabolism. Biochemistry 6, 1-5 (1967)

13. Wahren, J., Felig, P., Ahlborg, G., Jorfeldt, L.: Glucose metabolism during leg exercise in man. J. Clin. Invest. 50, 2715-2725 (1971)

14. Owen, O. F., Patel, M. S., Reichle, F. A., Block, B. S. B., Kreulen, T.H., Mozzoli, M. A.: Gluconeogenesis in the normal, diabetic, and cirrhotic human. In: Gluconeogenesis: Its Regulation in Mammalian Species, pp. 553-554 (1976)

Received: June 21, 1977,

and in revised form: December 19, 1977

Gerald M. Reaven, M. D.

Veterans Administration Hospital

111-M

3801 Miranda Avenue

Palo Alto, CA 94304

USA 